\section{Prostatakarzinom: Gute Erfahrung mit HIFU}

\begin{abstract}
Seit Jahren wird hoch intensiver fokussierter Ultraschall (HIFU) bei Männern mit lokalisiertem Prostatakarzinom angewandt. Aber es gibt nur kleine Studien - und nun eine Fallserie mit der bisher längsten Nachbeobachtungszeit.
\end{abstract}

$\mathrm{n}$ den vergangenen 15 Jahren wurden weltweit mehr als 25.000 an Prostatakrebs erkrankte Männer mit HIFU behandelt. In manchen Ländern, etwa in Italien, Frankreich und Großbritannien, wird die HIFU inzwischen von Fachgesellschaften bei bestimmten Patienten empfohlen - nicht jedoch in Deutschland.

Nun stellten Urologen um Roman Ganzer, Regensburg, ihre Erfahrungen mit der Methode vor. Zwischen 1997 und 2009 haben sie 538 Männer mit dieser Methode behandelt. Die Tumoren der meisten Teilnehmer waren im Frühstadium T1c und T2, also noch auf die Prostata begrenzt. Der Gleason-Score lag bei $75 \%$ der Patienten unter 6. Etwa $17 \%$ wurden nach den D'Amico-Kriterien der Hochrisikogruppe zugeordnet (Gleason-Score $\geq 8$, PSA $>20 \mathrm{ng} / \mathrm{ml}$ ). Im Mittel waren die Männer 68 Jahre alt.
Das Follow-up betrug im Durchschnitt acht Jahre (2,1-14 Jahre) - das längste, über das bisher berichtet wurde.

Biochemisch krankheitsfrei nach den Phoenix-Kriterien (Rezidiv bei Anstieg des PSA-Wertes um $2 \mathrm{ng} / \mathrm{ml}$ über den tiefsten PSA-Wert) überlebten fünf Jahre nach der HIFU-Behandlung insgesamt $81 \%$ der Behandelten, nach zehn Jahren immerhin noch $61 \%$. In den Subgruppen von Patienten, die einen Tumor mit niedrigem oder intermediärem Risiko nach D'Amico hatten, lagen die 5-Jahres-Werte sogar bei $88 \%$ und $83 \%$, nach zehn Jahren bei $71 \%$ und $63 \%$ - ein zufriedenstellendes Ergebnis, so die Ärzte.

In der Hochrisikogruppe waren die Ergebnisse allerdings schlechter. Nach fünf Jahren waren hier $48 \%$ der Patienten rezidivfrei, nach zehn Jahren war das aber nur noch bei $32 \%$ der Fall. Deshalb empfehlen Ganzer und seine Kollegen, die HIFU als Ersttherapie nicht bei Hochrisikopatienten mit einer Lebenserwartung von etwa zehn Jahren anzuwenden.

Die krebsspezifischen Überlebensraten lagen schließlich nach zehn Jahren in der Gruppe der Patienten mit niedrigem Risiko bei $100 \%$, bei Patienten mit intermediärem Risiko bei etwas mehr als $96 \%$. Und ohne Metastasen lebten nach diesem Zeitraum noch $99,6 \%$ beziehungsweise $94,3 \%$.

Fazit: Die transrektale Therapie mit HIFU beim lokalisierten Prostatakarzinom eignet sich für ältere Männer mit einer Lebenserwartung von wenigstens zehn Jahren. Das biochemisch definierte rezidivfreie Überleben beträgt der Studie zufolge nach fünf Jahren über $80 \%$. Aussagen über das Therapieergebnis bei jüngeren Patienten sind noch nicht möglich.

Peter Leiner

Ganzer R et al. Fourteen-year oncological and functional outcomes of high-intensity focused ultrasound in localized prostate cancer. BJU Int. 2013 Jan 28 [Epub ahead of print]

\section{Östrogenantagonist zur Prävention beim Prostatakarzinom?}

\section{Toremifen ist ein Modulator des Östrogenrezeptors. Eignet sich diese Substanz auch zur Prostatakrebsprävention bei Männern mit isolierter hochgradiger prostatischer intraepithelialer Neoplasie eignet.}

E in ine hochgradige prostatische intra- epitheliale Neoplasie (HGPIN) ist die Vorstufe eines Prostatakarzinoms. Männer mit isolierter HGPIN sind daher gute Kandidaten, um Strategien zur Chemoprävention zu testen. Toremifen ist ein oraler selektiver Östrogenrezeptormodulator, der für die Behandlung von fortgeschrittenem Brustkrebs zugelassen ist. In ersten Studien reduzierte die Substanz auch die Progression einer HGPIN zu einem Prostatakarzinom. Daher wurde nun in einer über drei Jahre laufenden Phase-III-Studie untersucht, ob Toremifen die Entwicklung eines Prostatakarzinoms bei Männern mit isolierter HGPIN verhindern kann.
1.590 Männer mit HGPIN, aber ohne Prostatakarzinom, erhielten drei Jahre lang randomisiert entweder $20 \mathrm{mg} / \mathrm{d}$ Toremifen oder Placebo. Die Teilnehmer waren 41 bis 87 Jahre alt (median 64 Jahre) und unterzogen sich jährlich einer Biopsie - bis zum Nachweis eines Tumors oder bis zum Studienende.

$34,7 \%$ der Männer in der Placebogruppe und 32,3\% der Männer in der Toremifengruppe entwickelten ein Prostatakarzinom. Das 3-Jahres-Überleben ohne Prostatakarzinom betrug $54,9 \%$ in der Placebogruppe und 59,5\% unter Toremifen, wobei der Unterschied zwischen beiden Gruppen nicht signifikant war ( $\mathrm{p}$ $=0,39$; log-rank-Test).
Bei der Analyse verschiedener Variablen wie Rasse, Alter, Prostatakarzinom in der Familienanamnese, Anzahl der Biopsien mit HGPIN, Vorhandensein einer atypischen mikroazinären Proliferation, Prostatagröße und PSA-Wert war in keiner Subgruppe eine Risikoreduktion durch Toremifen nachweisbar. Auch hinsichtlich der Nebenwirkungen und der Todesfälle gab es keine Unterschiede zwischen beiden Studienarmen.

Fazit: Toremifen konnte die Rate der nachgewiesenen Prostatakarzinome nicht verringern. Da Männer mit isoliertem HGPIN ein hohes Risiko für die Entwicklung eines Prostatakarzinoms haben, ist bei diesen Patienten eine Überwachung durch regelmäßige Prostatabiopsien erforderlich. Judith Neumaier

Taneja SS et al. Prostate cancer diagnosis among men with isolated high-grade intraepithelial neoplasia enrolled onto a 3-year prospective phase III clinical trial of oral toremifene. J Clin Oncol. 2013;31(5):523-9. 